\title{
Mechanical Properties and Nondestructive Testing of Advanced Materials
}

\author{
Yan Yang, ${ }^{1}$ Xing Chen, ${ }^{2}$ and Young Soo $\mathrm{Choi}^{3}$ \\ ${ }^{1}$ Key Laboratory of Manufacture and Test Techniques for Automobile Parts, Ministry of Education, Chongqing 400054, China \\ ${ }^{2}$ Department of Electrical and Computer Engineering, University of British Columbia, Vancouver, Canada V6T $1 Z 4$ \\ ${ }^{3}$ Department of Bioengineering, University of Washington, Seattle, WA 98195-5061, USA \\ Correspondence should be addressed to Yan Yang; yangyan@cqut.edu.cn
}

Received 15 December 2013; Accepted 15 December 2013

Copyright (C) 2013 Yan Yang et al. This is an open access article distributed under the Creative Commons Attribution License, which permits unrestricted use, distribution, and reproduction in any medium, provided the original work is properly cited.

Modern industry has been moving forward with unprecedented pace over past decades, while there is no doubt about the significant dedication by material science there. Of all the materials, advanced materials are becoming the driving source for extensive cutting-edge technologies, such as biomedical devices and wearable microelectronics. Generally speaking, the advanced material covers all the new materials, as well as existing materials but with modification for some specific applications, including ceramic materials, composites, metal alloys, and polymers.

Normally, any advanced materials should not be accepted by industry until their properties have been fully understood for the sake of reliability, especially for their mechanical properties that are of particular importance to engineering applications. With the advent of a huge number of new materials and continued evolution of other advanced materials, how to easily and quickly evaluate them remains challenging. Nondestructive testing presents the potential to be the solution for studying materials with both finance and time efficiency, which mainly relies on radiation, ultrasonic, and optical measurement methods.

This special issue thus focuses on mechanical properties and the nondestructive testing of advanced materials. Sixteen papers are invited herein to demonstrate the research on various advanced materials, such as shape memory alloy (SMA) and bamboo fibers. Many nondestructive testing methods and their implementation on advanced materials are introduced, including holography and laser-based lamb wave.
In the paper "Crack detection in single-crystalline silicon wafer using laser generated lamb wave," a noncontact and reliable measurement that was based on laser generated lamb wave has been used to detect crack existence in the brittle silicon wafer. Two modes of lamb wave were generated and detected, yielding accurate measurements. In the paper "Flexural vibration test of a beam elastically restrained at one end: a new approach for Young's modulus determination," a new vibration beam technique for the fast determination of the Young's modulus has been developed. The method is based on measuring the resonant frequency of flexural vibrations of a partially restrained rectangular beam. To validate this method, a number of industrial samples have been tested and the measured modulus was compared to the known values from the literature, showing good agreements. The work "Measurements of the characteristics of transparent material using digital holography" introduced a new method to optically measure characteristics of transparent materials by digital holography. The information of material surfaces was measured, and the characteristic was presented in $3 \mathrm{D}$ visualization with high fidelity which was validated by comparing it to the output from commercial counterpart. The paper "Deposition of low stress silicon nitride thin film and its application in surface micromachining device structures" talked about the work to deposit silicon nitride thin film with low stress and its application in surface micromachining. $\mathrm{SiH}_{2} \mathrm{Cl}_{2} / \mathrm{NH}_{3}$ gaseous ratio was adjusted to optimize the low stress recipe. The internal stress about $135 \mathrm{MPa}$ has been detected. 
In the research "Evaluation of ultrasonic nonlinear characteristics in heat-treated aluminum alloy (Al-Mg-Si-Cu)," the ultrasonic nonlinear parameters in the heat-treated aluminum alloy ( $\mathrm{Al}-\mathrm{Mg}-\mathrm{Si}-\mathrm{Cu}$ ) specimens have been measured. Its effectiveness to evaluate critical change in the elastic properties due to the thermal aging at high temperature was demonstrated. The paper "A study on contact fatigue performance of nitrided and TiN coated gears" discussed the effect of TiN coating on gear contact fatigue characteristics through contact fatigue experiment and gear rig test, which shows to be able to prolong the contact fatigue life of gears. In the study "Characteristics analysis and testing of SMA spring actuator," authors have discussed the shape memory alloy spring working as actuator and studied its displacement versus temperature by both theoretical model and the experimental method. The theoretical results are basically consistent to the experimental data. The paper "Analysis and testing of chain characteristics and rheological properties for magnetorheological fluid" presented to use digital holographic microscopy to measure magnetorheological (MR) fluid in different volume fraction of particles and under different magnetic field strengths. The chain model of dipole interaction of MR fluid was established, and the relationship between the shear stress and magnetic induction with particle volume fraction was obtained.

J. Ding et al. in the work "Mechanical performance evaluation of concrete beams strengthened with carbon fiber materials" used both finite element analysis and experimental validation to study the effect of carbon fibers in the reinforced plates. The finding revealed a big mechanical performance enhancement compared to that without carbon fibers. The work "Study of alloying process on 40Cr surface with electron beam after electroplated $\mathrm{Cr}$ layer" studied the precoating of electroplated chromium layer for improving surface properties of 40Cr. Microcracks have been detected by some measurement means, which can be eliminated by orthogonal experimental optimum process though. J. Shao et al. in the paper "Scaling analysis of the tensile strength of bamboo fibers using Weibull statistics" worked on scaling analysis of the mechanical properties of bamboo fibers by Weibull statistics. This study verified that the use of Weibull parameters from specimen testing can predict the strength distributions of fibers of longer gauge lengths. In the paper "Deformation properties and fatigue of bituminous mixtures," F. Schlosser et al. studied the deformation properties and fatigue of bituminous mixtures. Deformation properties can be used for empirical mixture design, and fatigue performance of asphalt mixtures in turn reflects the parameters of functional tests. Master curves express the properties of asphalt mixtures for various conditions and provide a time-efficient testing.

The work "Analysis and testing of MR shear transmission driven by SMA spring" described a novel transmission method of MR fluid in a cylindrical type that is driven by SMA spring. The transmit torque of MR fluid was controllable by the movement of coil-assembly with magnetic flux, which is actuated by SMA. By using this way, the transmission of mechanical torque can be controlled by temperature. In the paper "Vibration analysis of cylindrical sandwich aluminum shell with viscoelastic damping treatment," the vibration characteristics of a sandwiched cylindrical aluminum shell with viscoelastic damping treatment were investigated using layerwise theories. The cylindrical curved structure has been employed in many engineering applications, such as aircrafts, automobiles, ships, and other industrial machines. The results herein suggest that the sandwiched viscoelastic damping layer can effectively suppress vibration of cylindrical aluminum structure. Explosion pressure and compression pressure of internal combustion engine cylinder are two significant technical parameters affecting the total performance. By relying on microelectromechanical system (MEMS) technology, an intelligent pressure sensor with temperature coefficient compensation was demonstrated in the paper "Intelligent detector of internal combustion engine cylinder pressure and sensitivity temperature coefficient compensation." This technology exhibits the great practical value on internal combustion engine to test cylinder pressure, and the potential for volume manufacturing. The last paper in this special issue "An inverse method to reconstruct complete stiffness information of rubber bushing" discussed an inverse finite element method for parameters identification of rubber busing.

In summary, this special issue presents a broad range of topics relating to mechanical properties and the nondestructive testing of advanced materials. Valuable theoretical models and experimental testing methods have been introduced and discussed. Mechanical properties of different advanced materials have been studied, which suggests the promising features of them for wide applications. 

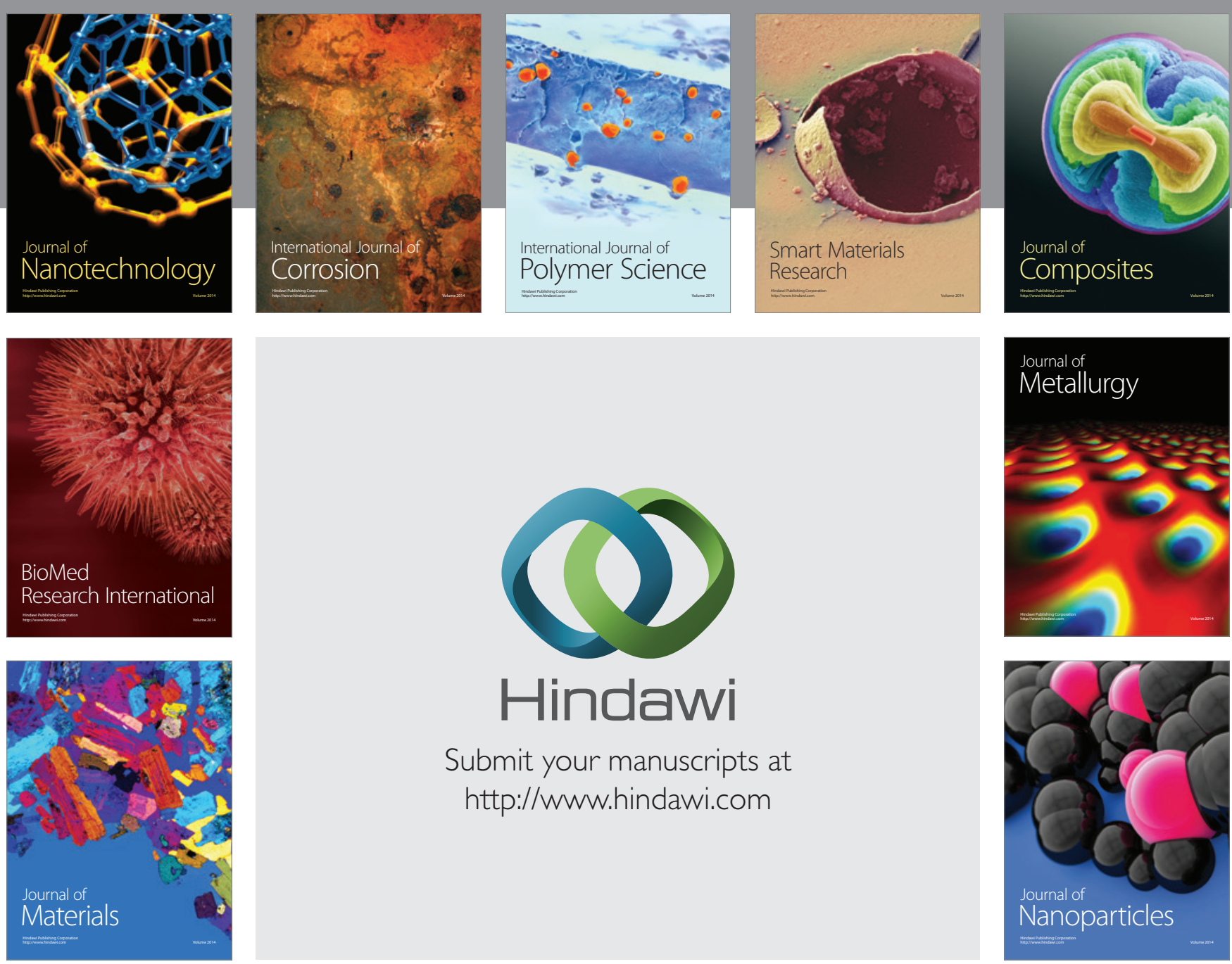

Submit your manuscripts at http://www.hindawi.com
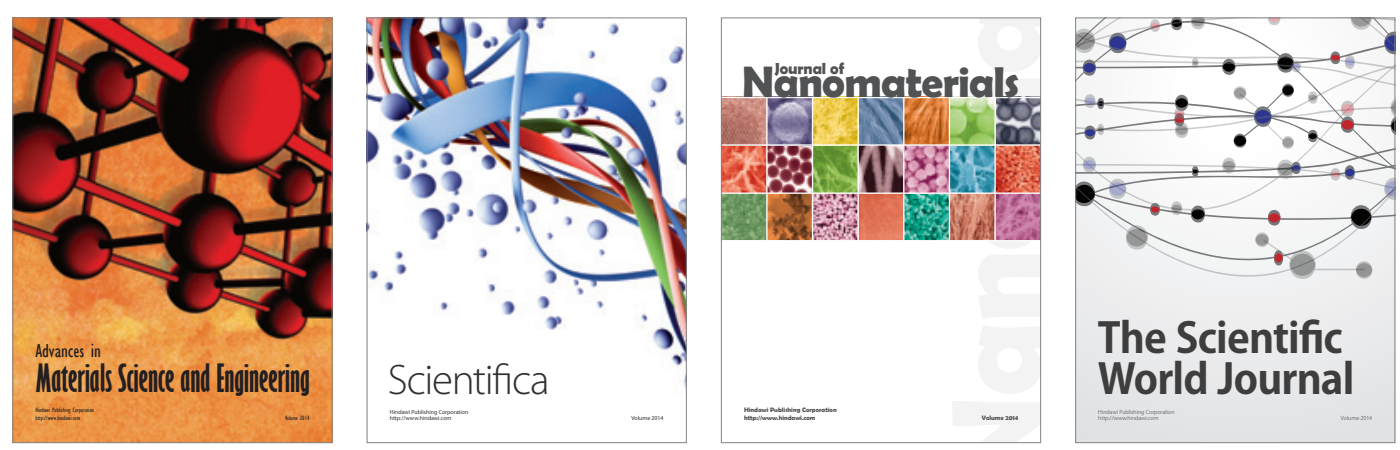

\section{The Scientific World Journal}
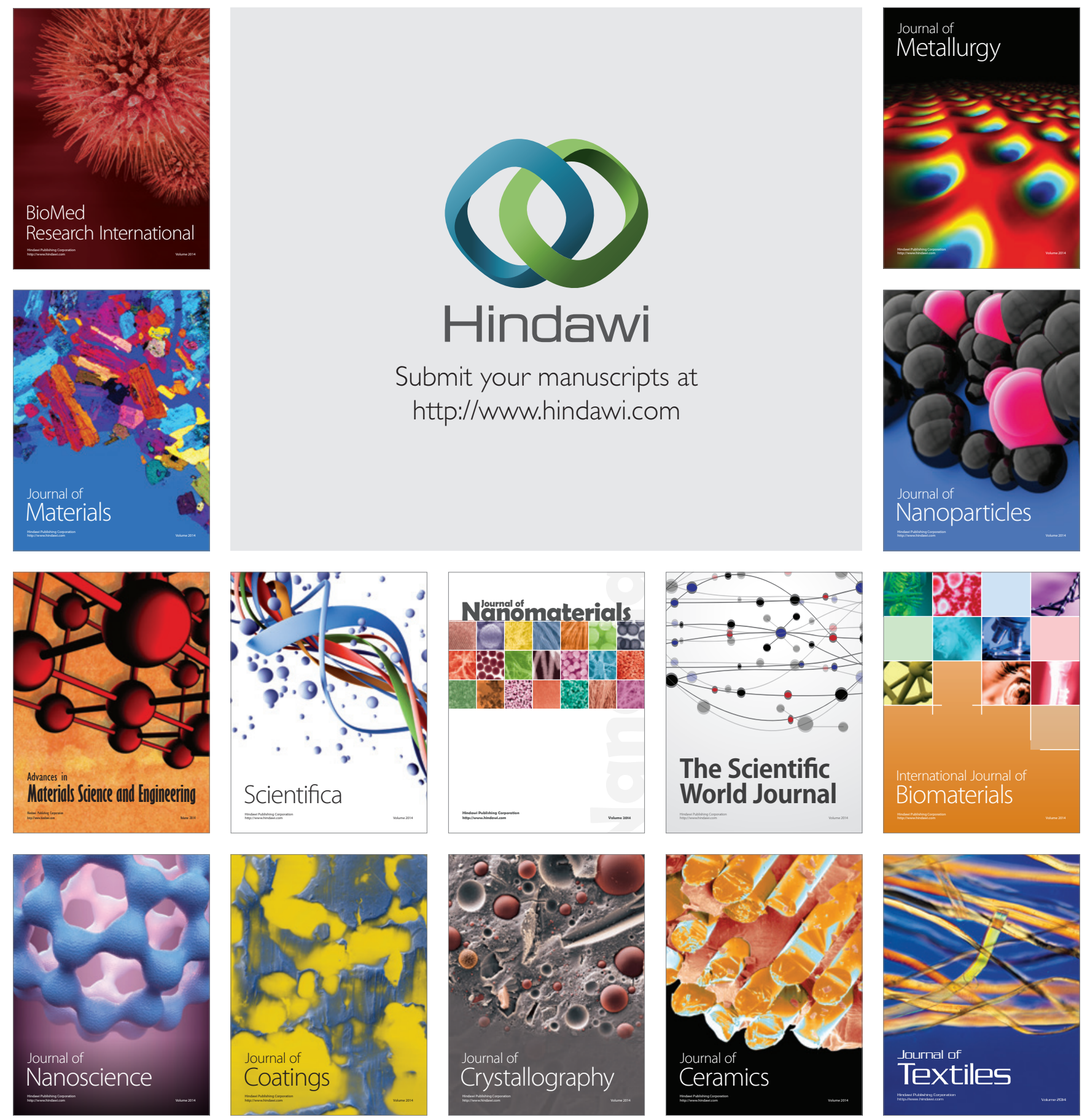